\title{
La Visitación de Saint- Jacques de Amberes: ¿Victor Wolfvoet o Simon de Vos?
}

\author{
The Visitation at Saint-Jacques Church in Antwerp: \\ Victor Wolfvoet or Simon de Vos?
}

\begin{abstract}
Matías Díaz Padrón ${ }^{1}$
Académie Royale d'Archéologie et d'Histoire de l'Art de Belgique Instituto Moll. Centro de investigación en pintura flamenca
\end{abstract}

\begin{abstract}
Resumen: El artículo trata de esclarecer la autoría propuesta y asumida a Victor Wolfvoet, desde finales del siglo XVII hasta hoy, de La Visitación de la iglesia de Saint-Jacques de Amberes restituyéndola a Simon de Vos. Al relato literario acompaña el documental y el análisis estilístico y técnico de la pintura, con obras análogas de este maestro como prueba comparativa a la atribución hoy propuesta.
\end{abstract}

Palabras clave: Pintura flamenca, Siglo XVII, Victor Wolfvoet, Simon de Vos, Amberes, Seguidores de Rubens.

Abstract: This article tries to clarify the authorship proposed to Victor Wolfvoet from the end of the $17^{\text {th }}$ century until nowadays, of the canvas of The Visitation at the church of Saint-Jacques in Antwerp. Here, it is proposed the painter Simon de Vos as its author. The documentary sources and the stylistic and technical analysis of the painting are taken as proofs to show the Simon de Vos authorship, comparing the painting with other similar works by the painter.

Key Words: Flemish Painting, $17^{\text {th }}$ century, Victor Wolfvoet, Simon de Vos, Antwerp, Rubens' followers.

Recibido: 9/05/2017

I motivo de estas líneas es el lienzo de la Visitación de la Virgen y San José a Santa Isabel y Zacarías situada en la primera capilla del deambulatorio del lado del evangelio de la iglesia de SaintJacques de Amberes (Fig. 1), poco antes de la tumba de Rubens, y tradicionalmente atribuida a Victor Wolfvoet. Hace unos años avancé otra

\footnotetext{
${ }^{1}$ orcid.org/ 0000-0002-5137-7583
}

(C) 2017 Philostrato. Revista de Historia y Arte 
atribución en una escueta nota al tratar un grupo de pintura en cobre y tabla de este pintor $^{2}$, proponiendo el nombre de Simon de Vos. La anterior atribución a Wolfvoet la fundamentaba el testimonio de Jacob de Wit, en la visita que realiza a mediados del siglo XVII a la iglesia de Santiago, donde la registra en el mismo lugar en el que hoy se encuentra: "Visitación por Víctor discípulo de Rubens"3. Alfred von Wurzbach dijo haber visto la firma y la fecha sobre el lienzo: "V. Wolfvoet, 1639"4, firma y fecha aceptadas sin crítica hasta ahora $^{5}$, y también con anterioridad por Théodore Van Lerius ${ }^{6}$.

La capilla de la Visitación de la iglesia de Santiago de Amberes se fundó el 5 de agosto de 1636 y consagró el 31 de mayo de $1640^{7}$, momento próximo a la fecha de la pintura. La iglesia, que se termina de construir en 1656, estaba dotada de una notable colección de pinturas (Fig. 2), por fortuna todas conservadas a pesar del paso de los siglos. Una visita desde el lado de la epístola hacia el del evangelio por el deambulatorio de la iglesia nos lleva a la capilla primera, con importantes pinturas de Hendrick van Balen y Jacob Jordaens. Ya en la segunda se encuentran otras de Gerard Seghers y Cornelio Schut. Especialmente interesante es la cuarta con la tumba de Rubens y su familia, erigida en 1643. El lienzo con la Virgen y santos de su mano en el altar de la capilla, nos recuerda el rostro de su joven mujer, Helena Fourment. Continuando con el itinerario, en las capillas quinta y sexta se reúnen obras de Jacob Jordaens y Peter Van Lint ${ }^{8}$. En la séptima capilla de este deambulatorio, al haber comenzado el recorrido desde el lado de la epístola, está la Visitación que nos ocupa atribuida a Víctor Wolfvoet, en cuyo rostro de la Virgen también se reconoce a Helena Fourment.

\footnotetext{
2 Matías Díaz Padrón, "Tres nuevos cobres restituidos a Víctor Wolfvoet, el más fiel seguidor de Rubens", Archivo Español de Arte, no 316, (2006), p. 407. Anuncié en este artículo la publicación del que ahora ocupa estas páginas. No siendo posible antes, por impedimento de la revista del CSIC a publicar obras de arte fuera del ámbito español, hecho que ha acontecido en estas últimas fechas. Es Víctor Wolfvoet pintor tratado en varias ocasiones en Archivo Español de Arte fundamentalmente, y en Tendencias del Mercado del Arte. Idem, "Dos cobres de Víctor Wolfvoet en el Museo San Carlos de México", Boletín del Seminario de Estudios de Arte y Arqueología, Universidad de Valladolid, no 65, (1999), p. 323; Idem. "Una adoración de los reyes de Víctor Wolfvoet en el coleccionismo madrileño", Tendencias del Mercado del Arte, no 18, (2008), p. 32; Idem, "Tres nuevos cobres de Víctor Wolfvoet con la Paz y la Guerra bajo la consigna de Rubens", Archivo Español de Arte, LXXXV, (2012), p. 88; Idem, "Una Andrómeda con Perseo y Pegaso en la lejanía de Víctor Wolfvoet atribuida a David Teniers en la galería Christie's de Nueva York", Tendencias del Mercado del Arte, no 53, (2012), p. 92; Idem, "Un Calvario de Víctor Wolfvoet en la colección Gerstenmaier", Tendencias del Mercado del Arte, no 68, (2014), p. 82. Recientemente Gregory Martin y Bert Schepers han tratado la obra de este pintor a partir de los estudios publicados en Archivo Español de Arte: Gregory Martin y Bert Schepers "Two Antwerp cabinets decorated by Victor Wolfvoet II", The Burlington Magazine, (2016), pp. 793-802.

3 Jacob de Wit, De Kerken van Antwerpen, (ed. J. de Bosschere, 1848, red. 1910), p. 36.

4 Alfred von Wurzbach, Niederländisches Kunstler Lexicon ans Gruma archivalister Farschungen, III, (Viena-Leipzig, 1906-1911), p. 899.

${ }^{5}$ Marie-Louise Hairs, Dans le Sillage de Rubens las peintres d'histoire anversois au XVII siècle, (Liège 1977), p. 221.

6 Théodore Van Lerius, Notice des œuvres d'art de l'église St. Jacques, (Amberes, 1855), p. 129.

7 Hairs, Dans le Sillage, p. 221, nota 30.

${ }^{8}$ Belgique, Les guides bleus, (Brussels, 1987), p. 199. La guía nos comunica estar ante pinturas de los más importantes maestros de este tiempo.
} 
Marie-Louise Hairs ve escasa fiabilidad en la firma del lienzo, por el medio siglo que distancia la cita de Von Wurzbach al no poder localizarla. No obstante, reconoce en el autor a un seguidor de Rubens que compara con la

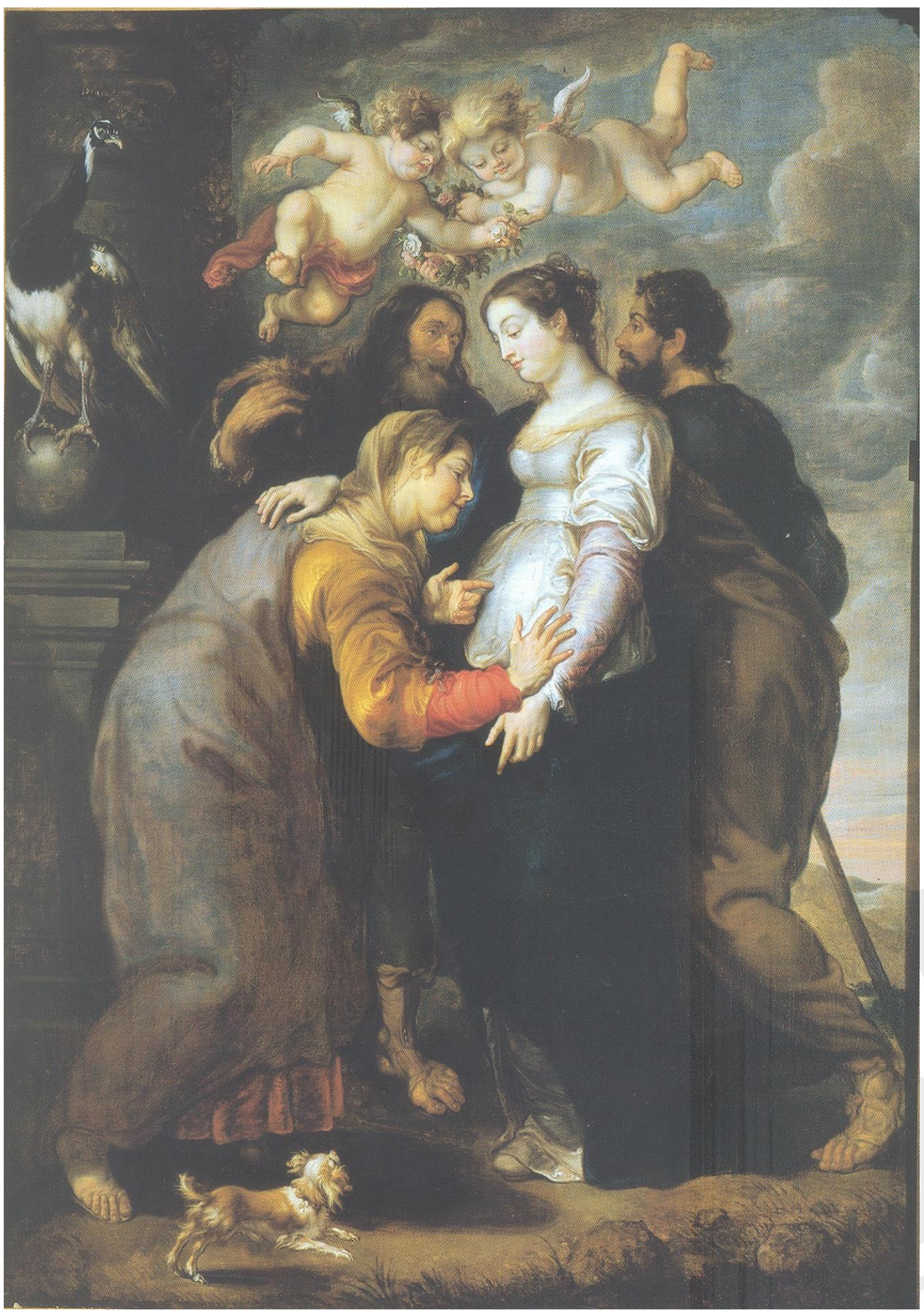

Fig. 1. Simon de Vos, Visitación de la Virgen, Amberes, iglesia de Saint-Jacques. 
Educación de María de Medici del Museo del Louvre (inv. no 1771), y diferencia de la escena de la Visitación del tríptico del Descendimiento de la Cruz de la catedral de Amberes (Fig. 3). Allí ve la discreta caricia del dedo de Isabel en el vientre de María y los esposos detrás. Aquí el grupo es más compacto y cerrado, con atribución tradicional a Victor Wolfvoet; una tradición impuesta por imperativos de la documentación literaria. Ningún testimonio visual se disponía en aquellas fechas para poder confrontarla con obras solventes de Victor Wolfvoet. Era muy escaso lo conocido y muy poco útil para un cotejo viable. El conocimiento de las obras de dicho maestro en el entorno de la galaxia de Rubens era muy reciente para dar un giro a la opinión tradicional.

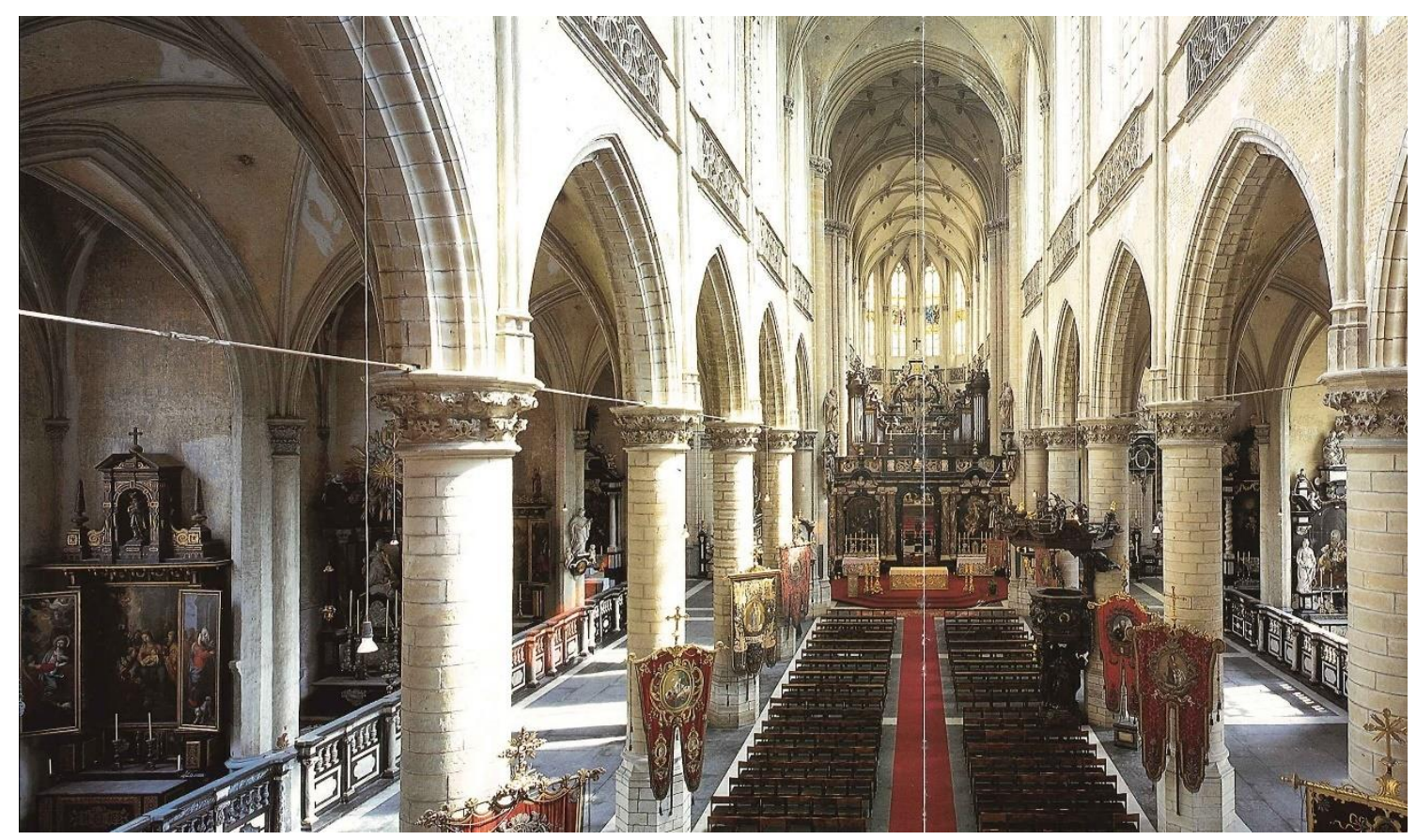

Fig. 2. Interior Iglesia de Saint-Jacques.

Tomando como punto de partida lo que escribía en $2006^{\circ}$, en el que se resumía el estado de la cuestión respecto a las opiniones en favor de Wolfvoet, expuestas líneas atrás, y las dudas esgrimidas por Hairs al no haber podido localizar la firma que citaban De Wit y Von Wurzbach sobre el lienzo, se unían mis propias sospechas para rechazar la tesis vigente. Proponemos ahora la autoría de Simon de Vos, a juzgar por el análisis de estilo y la técnica.

Simon de Vos es un importante pintor en el entorno de Rubens y Van Dyck, aunque no suficientemente estudiado. Gusta del alargamiento de las formas manieristas, los ritmos curvilíneos y las sombras envolventes, a distancia de

\footnotetext{
9 Díaz Padrón, "Tres sobres restituidos", p. 407.
} 
Victor Wolfvoet, juzgando lo hoy conocido de éste último. Este equívoco ha sido el obstáculo que ha impedido el estudio correcto de su obra.

La distinción y elegancia de la Virgen nos recuerdan más a Van Dyck que a Rubens. El sutil modelado en claroscuro de grises fundidos y negros contrasta con el rostro iluminado de la Virgen y blanco tisú de la túnica. Todo delata un estilo personal. Nada de rojos salvo un toque en el manto de Isabel y la gasa que envuelve la cintura a uno de los angelitos. Paradójicamente, el análisis del lienzo que hace Hairs, a quien muchos estudios debemos sobre los pintores del siglo XVII en Amberes, revela las características de Simon de Vos al juzgar el lienzo. Lejos de la dependencia industrial a Rubens de las obras de Victor Wolfvoet, recientemente localizadas por quien esto escribe, como ignoradas Hairs cuando escribía sobre el lienzo de Amberes.

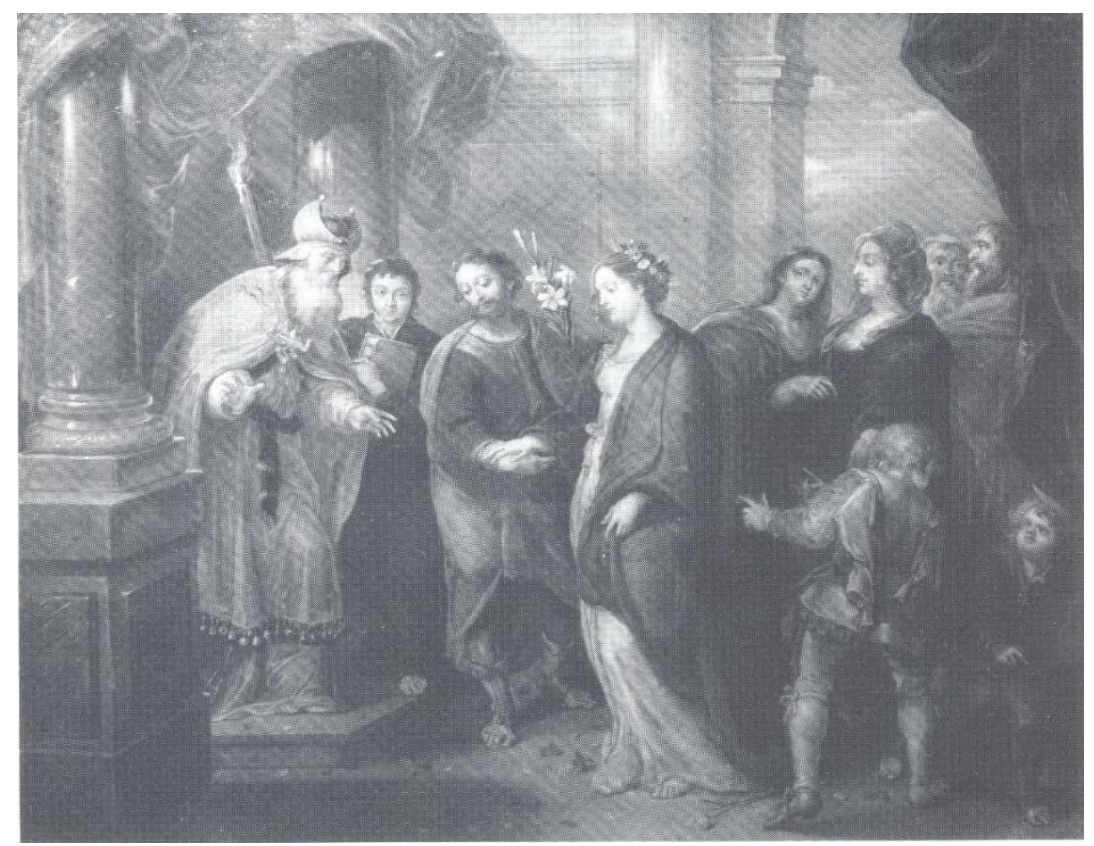

Fig. 4. Simon de Vos, Los desposorios de la Virgen, Lokeren

El autor de la Visitación asume sutiles ritmos ondulantes de personal sensibilidad, ajenos al servilismo de un copista. Tanto la técnica pictórica vibrante de Frans Francken II, a quien Victor Wolfvoet, por otro lado, también $\operatorname{copia}^{10}$, prisionero del pequeño formato y de una clientela distante de una élite exigente.

Interesante, por coincidir con el modelo de la Virgen de Simon de Vos que estudiamos, es la dibujada en Los desposorios de la Virgen de Lokeren ${ }^{11}$, erróneamente atribuido a Erasmus Quellinus (Fig. 4), y la Continencia de

\footnotetext{
10 Díaz Padrón, "Dos cobres de Victor Wolfvoet", p. 323.

11 Jean-Pierre De Bruyn, "Erasmus II Quellinus (1607-1678): addenda en corrigenda II", Koninklijk Museum voor Schone Kunsten Antwerpen, (1991), p. 166.
} 
Escipión de la Galería Drouot ${ }^{12}$. Por otro lado, una reducción de la Visitación de la iglesia de Amberes, con atribución a Rubens, conozco a través de los archivos del Cortauld Institute de Londres.

Es notoria la relación de la Visitación que restituimos a Simon de Vos con la de Rubens en el ala izquierda del Tríptico del Descendimiento de la catedral de Amberes realizada en 1611. La diferencia está en la inversión de los personajes de aquel encuentro. Los modelos de la Virgen y San José siguen igual actitud, con Santa Isabel tocando el vientre de María. Distinta es la gama cromática, la ondulación rítmica del dibujo y los dos angelitos en osado escorzo revoloteando en lo alto. El lienzo de Simon de Vos renuncia a la ostentosa arquitectura palaciega, lo que no quita que tribute al maestro en la utilización de las columnas conocidas columnas anilladas del pórtico que diseñó Rubens en su mansión antuerpiense. La luz que baña la figura de María contrasta con el resto de los reunidos en la penumbra del segundo plano.

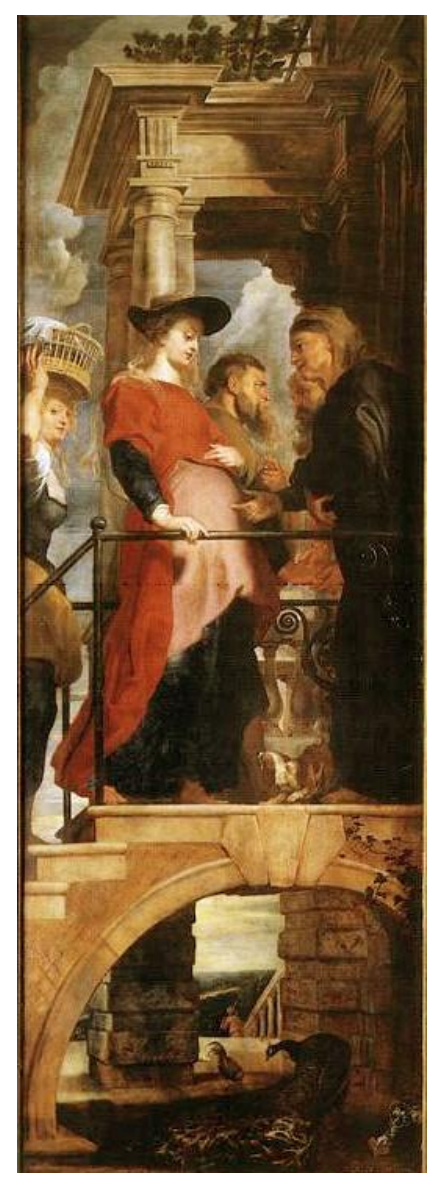

Fig. 3. P. P. Rubens, Visitación, Tríptico del Descendimiento, Amberes, catedral

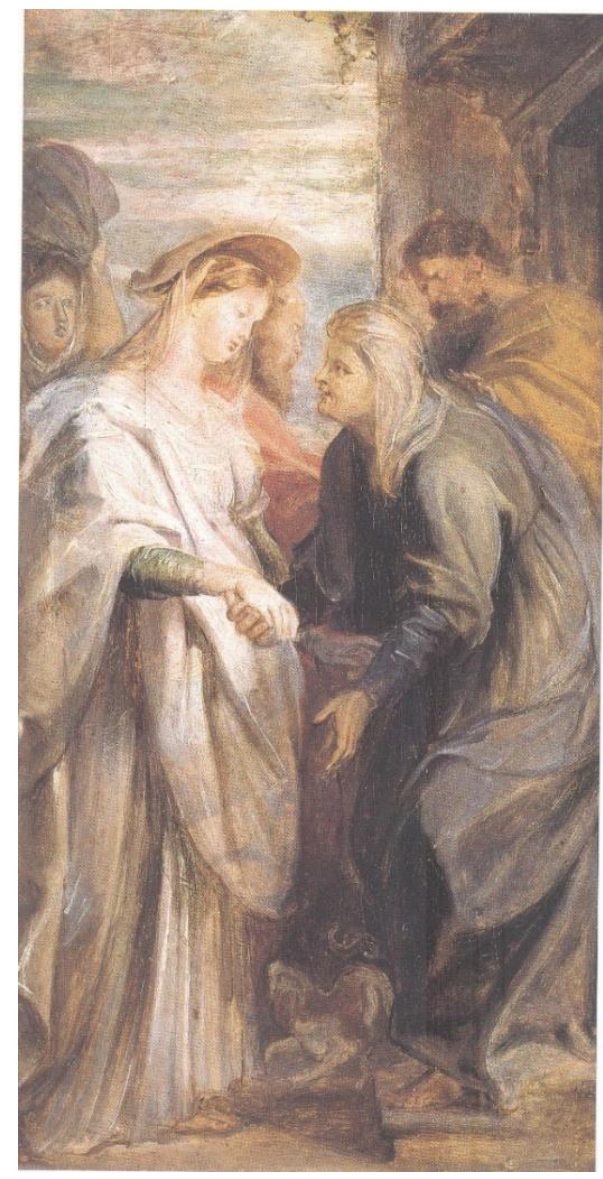

Fig. 5. P. P. Rubens, Visitación de la Virgen, Estrasburgo, Musée des Beaux-Arts

12 París, Drouot, 10-04-1996, no 8. 
El dibujo de Isabel con su dedo índice tocando el vientre de María del tríptico, es distinto a la versión de los marqueses de Ugena ${ }^{13}$, donde las dos mujeres se cogen las manos, pero sí coincide la inclinación reverencial de Isabel (Fig. 5). Tampoco las miradas frente a frente del lienzo de Rubens se repiten en la versión de Simon de Vos. Aquí la Virgen abraza a Isabel, mientras ésta toca y fija sus ojos en el vientre. La monumental distribución en triángulo no cambia. Se acusan diferencias en la concepción plástica del claroscuro, los colores dulces y la suave factura. Más profunda hondura sentimental presagia el rostro de Isabel fijando sus ojos en el vientre de su prima: "Es el hijo de Dios que va a salvar al mundo"14. La fuente remota de la Virgen en pie está en la Pudicitia que Rubens vio en Roma y dibujó en el Museo de Copenhague.

La reverencial inclinación de Isabel rompe con el plano frontal del modelo de Rubens, buscando un movimiento hacia el fondo del lado izquierdo. La luz

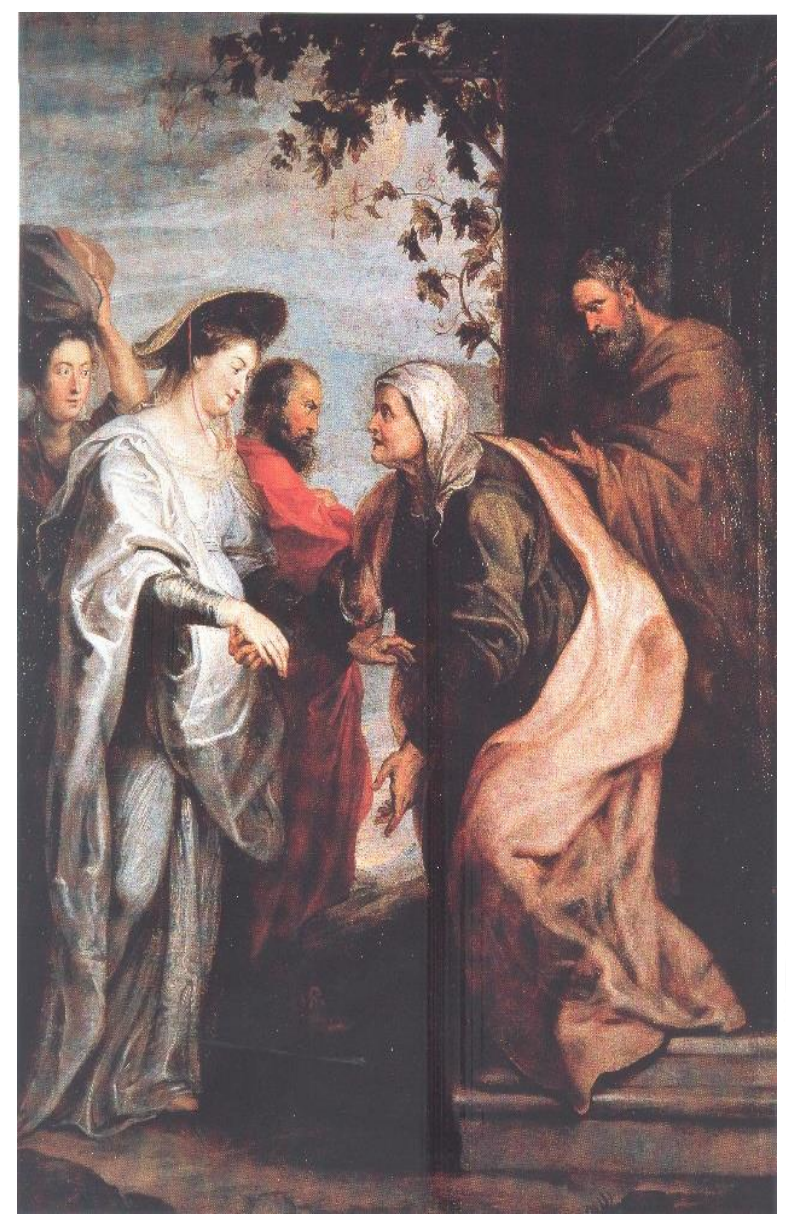

Fig. 6. P. P. Rubens, Visitación de la Virgen, antigua colección de Isabel María de la Cruz Ahedo, Marquesa viuda de Ugena.

\footnotetext{
13 Matías Díaz Padrón, La Visitación de la Virgen. Peter Paul Rubens (Madrid, 2005), p. 11; Ídem, "Una nueva Visitación de la Virgen de Peter Paul Rubens", Cuadernos de arte e iconografía, XIV, no 28, (2005), p. 256, nota 4.

14 Alfred Michiels, Histoire de la Peinture Flamande depuis ses débuts jusqu'en 1864, VIII, (Paris, 1869), p. 264.
} 
sobre San José y Zacarías se amortigua en favor del protagonismo de la Virgen. Simon de Vos no siguió al maestro al renunciar a los sirvientes que acompañaron a los protagonistas en la larga ruta. El gesto del semblante de Zacarías refleja los esfuerzos de comprensión que debe realizar al haber perdido la voz en castigo por sus dudas.

El imitar a Rubens en la composición no supuso la ruptura con el manierismo que subyace en la voluntad artística de Simon de Vos. El arabesco de los ritmos, la luz espectral y las sombras en penumbra son lo suyo, tanto como la lisa y esmaltada factura y distinción en los gestos. Características que persisten en las obras de gran formato del pintor fechadas hacia 1640. Éstas le van a dar más renombre, siguiendo los designios de la Contrarreforma. La tonalidad blanca del satén de la blusa y el amarillo de la túnica de Isabel son propios de su sentido del color.

El perrito a los pies es el símbolo de la fidelidad del matrimonio, en favor de la mujer ${ }^{15}$. Igual lectura tiene la yedra entrelazada en lo alto. En cuanto al pavo real sobre la bola de piedra y el plinto de traza renacentista, a la espalda de Isabel, es para Michiels (tratando la pintura con la atribución a Victor Wolfvoet), una intromisión sin sentido: "Yo no sé por qué se encuentra este pavo sobre la bola de piedra"16. Pero esta simbología no falta en la pintura flamenca de la Natividad. El pavo real es el símbolo de Juno velando por el matrimonio ${ }^{17}$. Los claveles de los angelitos simbolizan la felicidad ${ }^{18}$. Éstos llenan el espacio en la parte alta. Inexistentes en el tríptico, en el boceto del Museo de Bellas Artes de Estrasburgo ${ }^{19}$ (Fig. 6), y la colección de Isabel María de la Cruz Ahedo, marquesa viuda de Ugena ${ }^{20}$. Simon de Vos debió valerse de La Virgen y Santos que Rubens dispuso en el altar de su capilla funeraria. Es evidente la similitud con los dos angelitos en lo alto y el parecido

${ }^{15}$ Alciato, Emblemata, 1542, p. 1381; G. Ferguson, Sings \& Symbols in Christian Art, (Nueva York, 1966), p. 9.

16 Michiels, Histoire de la Peinture, p. 263.

17 Valeriano, Hieroglyphica...Ioanis Pierii Valeriani, Bale, (ed. Príncipe, 1556), XXIII, "pavo concordia"; G. De Tervarent, Attributs et Symboles dans l'art profane 1450- 1600. Dictionnaire d'un langage perdu, (Geneve, 1958), p. 298.

${ }^{18}$ Ferguson, Sings \& Symbols, p. 34.

19 Peinture Flamande et hollandaise XVe-XVIIIe siècle, (Strasbourg, 2009), p. 132, a las observaciones personales de J. Hubrecht, tratando del lienzo publicado en colección española, posiblemente a través de fotografías, habría que añadir algunas referencias que no conoce en estudio de 2005. Díaz Padrón, "Una nueva Visitación", p. 256.

20 En 2005 se pudo precisar la historia externa de la pintura. A mediados del siglo XVII estaba en la colección de Doña Isabel María de la Cruz Ahedo, marquesa de Ugena. La testamentaria de 1747 dice: "Primeramente una pintura de la Visitación con nuestra señora y Santa Isabel, de dos varas de alto y más de media de ancho, marco dorado y tallado, original de Rubens en 9000 reales vellón". Es la pintura que alcanza mayor tasación en el inventario (Archivo Histórico de Protocolos, no 15.792 y 15.798 . Inventario de los bienes al fallecer don Juan Francisco de Goyeneche, caballero de la orden de Santiago, marqués de Ugena y Torrejoncillo, del consejo de su majestad y mayordomo de la reina nuestra señora.) Díaz Padrón, "Una nueva visitación", p. 274, nota 10. Tras su venta, quedó en manos de don Antonio de Castro, que la compró en 3000 reales: "Vienes que había en la casa que se vendieron... en don Antonio de Castro una pintura de la Visitación, de Rubens, en 3000 reales de vellón", documentos publicados por Hernando Álvarez. E. Hernando Álvarez, "La colección de pinturas de don Juan Francisco de Goyeneche y su esposa", Archivo Español de Arte, 250, (1990) p. 335; Díaz Padrón, "Una nueva Visitación", p. 256 y p. 274, nota 11. 
del rostro de Helena Fourment. El pavo real lo encuentro de nuevo en la tabla de la Educación de Jesús con la Virgen y Santa Ana, firmada por Simon de Vos en colección privada española, (Fig. 7) de importante interés para la autoría del lienzo que estudiamos en la iglesia de Saint-Jacques.

El tema de la Visitación fue muy divulgado desde el siglo XV y XVI en la pintura flamenca, con el encuentro, el abrazo y la genuflexión de Isabel. La Virgen baja la cabeza con sumiso decoro y dignidad. El pintor fue fiel al escrito del libro I de San Lucas:

"En aquello días se puso María en camino y con presteza fue a la montaña, y entró en casa de Zacarías y saludó a Isabel. Así que Isabel oyó el saludo de María, exultó el niño en su seno e Isabel se llenó del Espíritu Santo y exclamó: Bendita tu entre las mujeres y bendito el fruto de tu vientre. ¿De dónde a mí que la madre de mi señor venga a mí? Porque así que sonó la voz de tu salutación en mis oídos exultó de gozo el niño en mi seno. Dichosa la que ha creído que se cumplirá lo que se le ha dicho de parte del Señor. Dijo María: Mi alma, mi grandeza, el Señor. Y exulta de júbilo mi espíritu en Dios, porque ha mirado la humildad de su sierva" (LC., 1, 39-48).

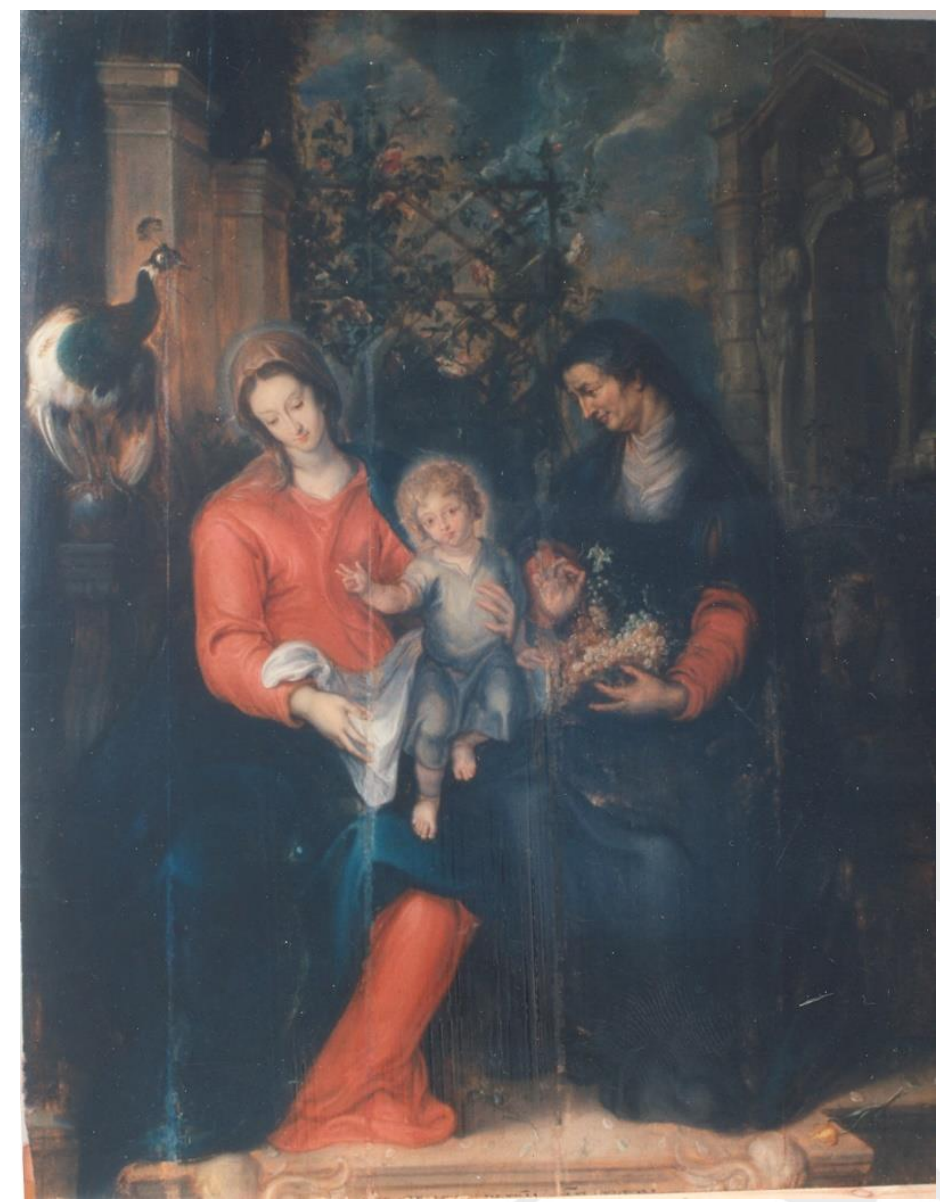

Fig. 7. Simon de Vos, Educación de Jesús con la Virgen y Santa Ana, colección privada, Madrid 
A pesar de la influencia de Rubens y Van Dyck, Simon de Vos sigue fiel a una personalidad que no ha sido valorada en su justa medida. Es pintor merecedor de una mayor atención. Francine-Claire Legrand lo estudió entre los pintores de género ${ }^{21}$, pero los lienzos de gran formato le dan un lugar dentro del capítulo de los maestros de mayor renombre. En este apartado habría que colocar la Visitación de la iglesia de Saint-Jacques de Amberes.

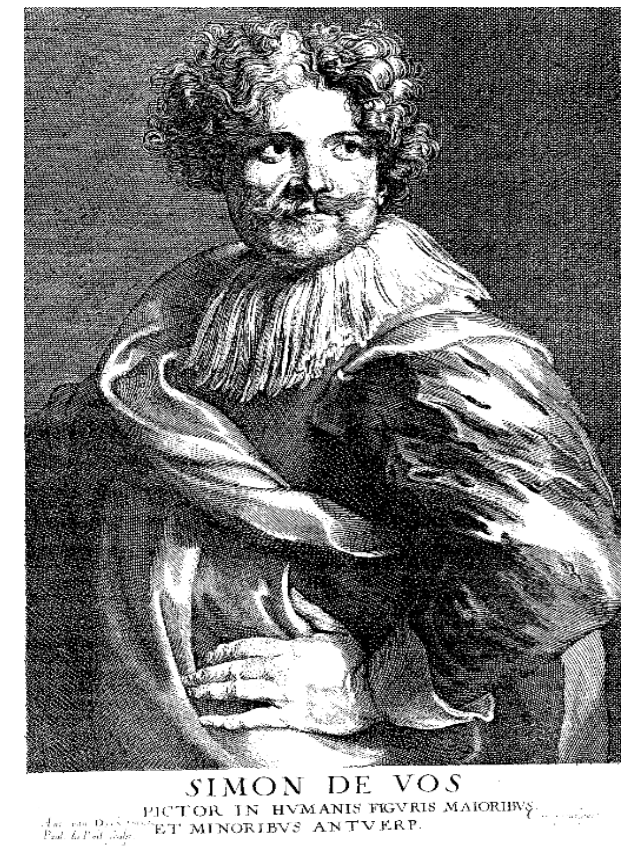

Fig. 8. A. Van Dyck, Retrato de Simon de Vos, grabado por Paul du Pont

Van Dyck lo inmortalizó en un retrato de su Iconografía junto a los maestros más laureados de su tiempo (Fig. 8) ${ }^{22}$. Alfred Michiels lo inscribe entre los más afamados discípulos de Van Dyck, "de él ha tomado su estilo y, aunque discípulo de Rubens -escribe- se sintió más atraído por el gran discípulo de su maestro"23. De gran formato son los lienzos de La Creación que exportó a la catedral de Sevilla en su tiempo ${ }^{24}$. Algo llegó a Valencia 25 , y a las

\footnotetext{
${ }^{21}$ Francine-Claire Legrand, Les peintres flamands de genre au XVIIème siècle, (Brussels, 1963), pp. 5762.

${ }^{22}$ F. W. H. Hollstein, The new Hollstein Dutch \& Flemish etchings, engravings and woodcuts 1450-1700. Anthony Van Dyck, II, Amsterdam, 2012, p. 181; "Simon de Vos. Pictor in Hvmanis Figvris Maioribvs et minoribvs antverp", p. 183; Under van Dyck's name: Paul. Du Pont. Sculp. Amsterdam (RP-P-OB-33.298).

${ }^{23}$ Alfred Michiels, Van Dyck et ses Élèves, (Paris, 1882), p. 564.

24 Matías Díaz Padrón, "Simon de Vos en la catedral de Sevilla", Archivo Español de Arte, no 192, (1975), p. 397.

${ }^{25}$ Felipe Vicente Garín Llombart, "Simon de Vos y Van der Poel en el Museo de Bellas Artes", Archivo de Arte Valenciano, 41, (1970), p. 31.
} 
Américas $^{26}$. Otras, en gran número, llegan a Polonia ${ }^{27}$. No olvidamos, como prueba de la estimación que sintió Rubens, tener entre su colección una obra suya con la historia del Hijo Pródigo ${ }^{28}$. La inclinación al gran formato por influencia de Rubens se fija entre 1630-1640, cronología en sintonía con la Visitación de Saint-Jacques de Amberes.

Michiels al analizar el estilo de la Visitación atribuida a Victor Wolfvoet, escribe lo propio del estilo y técnica de Simon de Vos. Toma noticias de Cornelis de Bie, para quien la capacidad de Simon de Vos fue igual para el gran formato como para el pequeño. Pero, paradójicamente, dice no encontrar pinturas suyas en Amberes, ni en ningún otro lugar ${ }^{29}$. Interesante para su relación con la iglesia de Saint-Jacques, donde se ignora su nombre, al hablar de su Visitación, eso ayuda a ésta. Entregó una rica donación para su fábrica, al igual que hizo para la iglesia-catedral de Nuestra Señora, con mayor generosidad ${ }^{30}$, prueba de su capacidad económica y su magnificencia.

\footnotetext{
26 Diego Angulo Íñiguez, "Un cuadro de Simón de Vos en Lima", Archivo Español de Arte, 16, (1943), p. 414.

27 Janina Michalkowa, "The tableaux de Simon de Vos dans les collections polonaises", Bulletin du Musée National de Varsovie, XVII, (1977), pp. 1-21.

28 "L'Enfant prodigue de Symon de Vos". Julius Samuel Held, "Artis Pictoriae Amator: An Antwerp Art Patron and His Collection", Gazette des Beaux-Arts, 50, (1957), pp. 53-84, fig. 4., no 26; vid. J.M. Muller, Rubens: The Artist as Collector, (Princeton, 1989), p. 136; Kristin Lohse Belkin, Fiona Healy, A House of Art Rubens as Collector, (Rubenianum, Antwerp, 2004), p. 332, no 241.

${ }^{29}$ Michiels, Histoire de la Peinture, p. 302. Entre la escasa obra de Víctor Wolfvoet que conoce, cita la Cabeza de Medusa del Museo de Dresde, como original del Víctor Wolfvoet el viejo. Hoy sabemos que es del hijo, elogiando su valor (Michiels, Histoire de la Peinture, p. 264). Añade un Paso del mar Rojo firmado, que pensamos que sea también del hijo y copiando posiblemente un original de Frans Francken II (Vid. Díaz Padrón, "Dos cobres de Víctor Wolfvoet", p. 323).

30 Michiels, Histoire de la Peinture, p. 301.
} 
Bibliografía:

Alciato 1542: Alciato, Emblemata, 1542.

Angulo Íñiguez 1943: Diego Angulo Íñiguez, "Un cuadro de Simón de Vos en Lima", Archivo Español de Arte, 16, (1943), p. 414.

Belgique 1987: Belgique, Les guides bleus, (Brussels, 1987).

De Bruyn 1991: Jean-Pierre De Bruyn, "Erasmus II Quellinus (1607-1678): addenda en corrigenda II", Jaarboek Koninklijk Museum voor Schone Kunsten Antwerpen, (1991), pp. 157-198.

De Tervarent 1958 : G. De Tervarent, Attributs et Symboles dans l'art profane 1450- 1600. Dictionnaire d'un langage perdu, (Geneve, 1958).

De Wit 1910: Jacob de Wit, De Kerken van Antwerpen, ed.J. de Bosschere, 1910 (1848).

Díaz Padrón 1975: Matías Díaz Padrón, "Simon de Vos en la catedral de Sevilla", Archivo Español de Arte, no 192, (1975), pp. 397-402.

Díaz Padrón 1999: Matías Díaz Padrón, "Dos cobres de Víctor Wolfvoet en el Museo San Carlos de México", Boletín del Seminario de Estudios de Arte y Arqueología, Universidad de Valladolid, no 65, (1999), pp. 323-328.

Díaz Padrón 2005: Matías Díaz Padrón, "Una nueva Visitación de la Virgen de Peter Paul Rubens", Cuadernos de Arte e Iconografía, XIV, n 28, (2005), pp. 255-279.

Díaz Padrón 2005: Matías Díaz Padrón, La Visitación de la Virgen. Peter Paul Rubens, (Madrid, 2005).

Díaz Padrón 2006: Matías Díaz Padrón, "Tres cobres restituidos a Víctor Wolfvoet, el más fiel seguidor de Rubens", Archivo Español de Arte, no 316, (2006), pp. 403-411.

Díaz Padrón 2008: Matías Díaz Padrón, "Una adoración de los reyes de Víctor Wolfvoet en el coleccionismo madrileño", Tendencias del Mercado del Arte, no 18, (2008), pp. 32-33.

Díaz Padrón 2012: Matías Díaz Padrón, "Tres nuevos cobres de Víctor Wolfvoet con la Paz y la Guerra bajo la consigna de Rubens", Archivo Español de Arte, LXXXV, (2012), pp. 75-94.

Díaz Padrón 2012: Matías Díaz Padrón, "Una Andrómeda con Perseo y Pegaso en la lejanía de Víctor Wolfvoet atribuida a David Teniers en la galería Christie's de Nueva York", Tendencias del Mercado del Arte, no 53, (2012), pp. 92-94.

Díaz Padrón 2014: Matías Díaz Padrón, "Un Calvario de Víctor Wolfvoet en la colección Gerstenmaier", Tendencias del Mercado del Arte, nº 68, (2014), pp. 82-84. 
Ferguson 1966: G. Ferguson, Sings \& Symbols in Christian Art, (New York, 1966).

Garín Llombart 1970: Felipe V. Garín Llombart, "Simon de Vos y Van der Poel en el Museo de Bellas Artes", Archivo de Arte Valenciano, 41, (1970), pp. 3132.

Hairs 1977: Marie-Louise Hairs, Dans le Sillage de Rubens las peintres d'histoire anversois au XVII siècle, (Liège, 1977).

Held 1957: Julius Samuel Held, "Artis Pictoriae Amator: An Antwerp Art Patron and His Collection", Gazette des Beaux-Arts, 50, (1957), pp. 53-84.

Hernando Álvarez 1990: E. Hernando Álvarez, "La colección de pinturas de don Juan Francisco de Goyeneche y su esposa", Archivo Español de Arte, 250, (1990) pp. 331-335.

Legrand 1963: Francine-Claire Legrand, Les peintres flamands de genre au XVIIème siècle, (Brussels, 1963).

Lohse Belkin y Healy 2004: Kristin Lohse Belkin y Fiona Healy, A House of Art Rubens as Collector, Rubenianum, (2004).

Martin y Schepers 2016: Gregory Martin y Bert Schepers "Two Antwerp cabinets decorated by Victor Wolfvoet II", The Burlington Magazine, 158, no 1363, (2016), pp. 793-802.

Michalkowa 1977: Janina Michalkowa, "The tableaux de Simon de Vos dans les collections polonaises", Bulletin du Musée National de Varsovie, XVII, (1977), pp. 1-21.

Michiels 1869: Alfred Michiels, Histoire de la Peinture Flamande depuis ses débuts jusqu'en 1864, VIII, (Paris, 1869).

Michiels 1882: Alfred Michiels, Van Dyck et ses Élèves, (Paris, 1882).

Müller 1989: J.M. Müller, Rubens: The Artist as Collector, (Princeton, 1989).

New Hollstein 2012: F. W. H. Hollstein, The new Hollstein Dutch \& Flemish etchings, engravings and woodcuts 1450-1700. Anthony Van Dyck, II, (Amsterdam, 2012).

Strasbourg 2009: Peinture Flamande et hollandaise XVe-XVIIIe siècle, (Strasbourg, 2009).

Valeriano, Hieroglyphica...Ioanis Pierii Valeriani, Bale, (ed. Príncipe, 1556).

Van Lerius 1855: Théodore Van Lerius, Notice des œuvres d'art de l'église St. Jacques, (Anvers, 1855).

Wurzbach 1906-1911: Alfred von Wurzbach, Niederländisches Kunstler Lexicon ans Gruma archivalister Farschungen, III, (Viena-Leipzig, 19061911). 